\title{
Statistical Diagnosis Method of Conductor Motions in Superconducting Magnets to Predict their Quench Performance
}

\author{
P. Pugnat, B. Khomenko, A. Rijllart, S. Sanfilippo and A. Siemko
}

\begin{abstract}
Premature training quenches are usually caused by the transient energy released within the magnet coil as it is energised. Two distinct varieties of disturbances exist. They are thought to be electrical and mechanical in origin. The first type of disturbance comes from non-uniform current distribution in superconducting cables whereas the second one usually originates from conductor motions or micro-fractures of insulating materials under the action of Lorentz forces. All of these mechanical events produce in general a rapid variation of the voltages in the so-called quench antennas and across the magnet coil, called spikes. A statistical method to treat the spatial localisation and the time occurrence of spikes will be presented. It allows identification of the mechanical weak points in the magnet without need to increase the current to provoke a quench. The prediction of the quench level from detailed analysis of the spike statistics can be expected.
\end{abstract}

Index Terms-Conductor motions, Quench training, LHC, Spikes, Statistics, Superconducting magnets.

\section{INTRODUCTION}

$I^{2}$ 1998, CERN launched in European industry the fabrication of six final design main dipole prototypes for the Large Hadron Collider (LHC). Four cold masses have so far been fully assembled and already tested at CERN. A presentation of the quench performance and the field quality of these prototypes is given in [1]. These magnets operate at $1.9 \mathrm{~K}$ and are designed to have their superconducting cable limit at $9.76 \mathrm{~T}$. Like most large-scale magnets, the LHC dipoles exhibit training quenches i.e. a progressive improvement of the field level reached after repeated quenching. This type of premature quenches is mainly due to conductor motions or epoxy resin cracking. Both of these effects can lead to a local temperature rise exceeding the critical temperature of the superconducting state.

The pick-up coil technique developed at CERN [2] was

Manuscript received September 18, 2000

Authors are with the European Laboratory for Nuclear Research, CERN, CH-1211, Geneva 23, Switzerland.

P. Pugnat, (telephone: +4122-767-9533, e-mail: Pierre.Pugnat@cern.ch).

B. Khomenko, (telephone: +4122-767-7926, e-mail:

Boris.Khomenko@cern.ch).

A. Rijllart, (telephone: +4122-767-8593, e-mail: Adriaan.Rijllart@cern.ch)

S. Sanfilippo, (telephone: $+4122-767-8018$, e-mail:

Stephane.Sanfilippo@cern.ch)

A. Siemko, (telephone: +4122-767-8649, e-mail: Andrzej.Siemko@cern.ch). originally used to detect the local magnetic field distortion provoked by the current redistribution during a quench. The localisation of premature quenches using pick-up coils also called Quench-Antenna (QA), and Voltage-Taps (VT) are in daily use to improve the magnet design and fabrication [1]. Using the same techniques signals occurring well before quenches can also be studied. The rapid variations recorded in voltages coming from QA and VT called spikes, can in general be interpreted as due to conductor motions or epoxy resin cracking. The location and the time distribution of such precursors to the quench can give precious information concerning the mechanical stability of the coils. In order to perform a statistical study, dedicated tools for data treatment were developed.

In this article, the geometry of the pick-up coils used to localise quenches and precursors will be presented first. Then the development of software to identify and discriminate mechanical events will be summarised. Some examples of results coming from power tests of LHC prototype dipoles, analysed with a first software version will be presented. The relevant parameters of the statistical events recorded will be discussed from the point of view of the mechanical stability of the coil. Finally, the exploitation of such development for the analysis of the performance of LHC magnets will be presented.

\section{EXPERIMENTAL}

\section{A. Voltage-Taps and Quench-Antennas techniques}

Ceramic shafts composed of 13 identical segments were built in order to cover the two apertures of 15-meter long LHC dipoles with pick-up coils [3]. Segments are made of a ceramic tube containing three identical tangential search coils each having a length of $1.116 \mathrm{~m}$ (Fig. 1). These shafts are mechanically connected on the test bench to a twin rotation unit. They are also used to measure harmonic components of the magnetic field of the dipoles [1],[3]. A dedicated study of vibration modes of the shafts connected on the test bench has been performed. No mechanical vibrations at frequencies higher than $2 \mathrm{kHz}$ were recorded.

The acquisition system records signals both from VT and QA. It is triggered by the quench detectors used for the magnets protection. Several compensation schemes, which connect pick-up coils in pairs are used to cancel the noise coming mainly from the power converter. The maximum sampling frequency of the multi-channel transient recorder is $50 \mathrm{kHz}$. The transient recorder can be used in multi-event 
mode to record spikes that occur during the current ramping up to a quench. To record a maximum number of pre-quench spikes with a sufficient time resolution, $5 \mathrm{kHz}$ recording was used and the pre-trigger time was set to $9 \mathrm{~s}$.

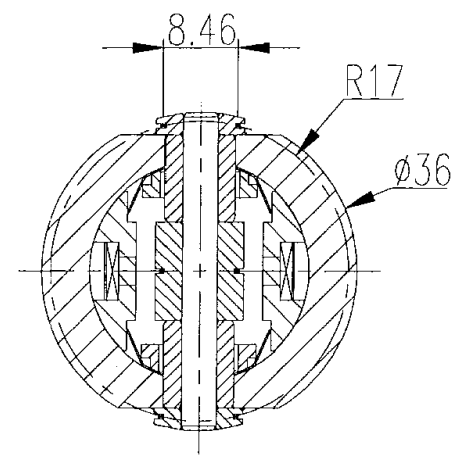

Fig. 1. Cross-section of a segment of a Quench-Antenna with the main dimensions. Tangential coils can be seen on the top, middle and bottom of the drawing.

\section{B. Software development}

Example of signals interpreted as a conductor motion

A typical example of signals interpreted as coming from conductor motion is given in Fig. 2. They were recorded during the current ramping up on one of the full-scale LHC prototype magnets tested.

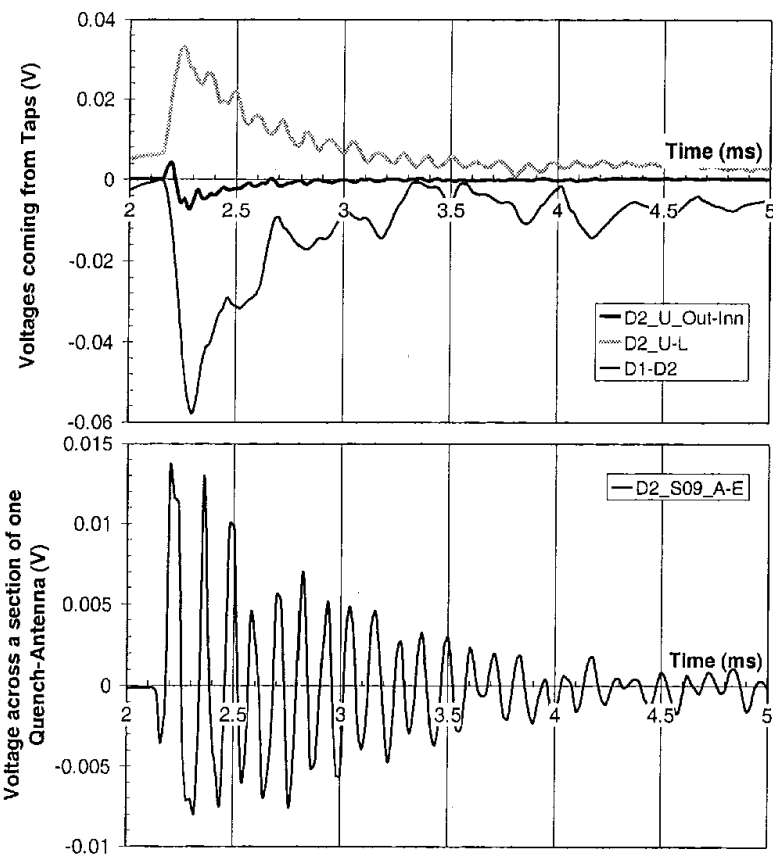

Fig. 2. Example of signals coming from Voltage-Taps and QuenchAntenna and interpreted as a conductor motion inside the magnet coil.

Quench-Antenna voltages oscillate at a main frequency of $8.9 \mathrm{kHz}$ with strong dumping, whereas Voltage-Taps give signals with well pronounced maximum or minimum superimposed with the oscillation. The shape difference between both types of signals mainly comes from different filtering applied. Typical spike duration lies in the range $0.1-10 \mathrm{~ms}$. The axial segmentation into 13 sections of QA as well as the layout of the VT allow the localisation of spikes in a similar way as quenches. For example in Fig. 2, the spike voltage difference between aperture, V(D1-D2), is negative which means in our convention, that the spike appeared in aperture 2. Successively repeating this reasoning, the spike shown can be fully localised inside dipole 2 at axial section 09 in the inner layer of the upper pole.

\section{Example of a collection of signals interpreted as conductor} motions

An example of a collection of spikes recorded during $70 \mathrm{~ms}$ from sections of the aperture 2 is shown in Fig. 3.

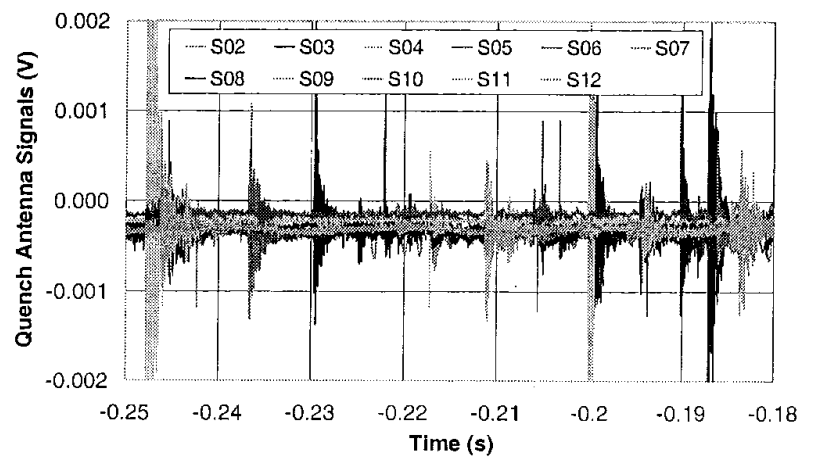

Fig.3. Collection of spikes recorded $70 \mathrm{~ms}$ before a quench from QA sections of one aperture

The number of "mechanical events" which can be counted is around 30. One of the major problems encountered in software development is to count the number of independent events. Indeed, spikes propagate along the magnet axis with a measured velocity of $1-2 \mathrm{~km} / \mathrm{s}$ (the velocity of sound inside the conductor while cold) as well as between apertures.

\section{Signals treatment}

Programs dedicated to analysing spikes have been developed using LabVIEW $^{\circledR}$ on a Sun ${ }^{\circledR}$ workstation. Three main stages can be put forward. The first one concerns the extraction of characteristic signals (Fig. 2) after noise rejection (identification). The second one is related to the association of spikes coming from the same event (globalisation). The third stage is the parameterisation of individual spikes to calculate all statistical parameters needed to characterise general features and evolution of the mechanical activity of magnets during tests.

Apart from methods of association, both channel groups for QA and VT were treated identically. So far, more attention was paid to QA channels which record more events than VT and provide information about spike distribution along the axis of the magnet. As two compensation schemes are used, every QA channel provides a differential measurement either inside some magnet section or between different sections. To avoid ambiguities and problems of redundancy, only the first 
kind of QA channels was treated for the moment. The channels recorded at $5 \mathrm{kHz}$ during $9 \mathrm{~s}$ before the quench, were scanned independently to detect spike signals as narrow peaks or oscillations exceeding the level of background noise characterised within each channel by its standard deviation $\sigma$. The $5 \sigma$ criterion was used in the data given in this study but this number can be used as a free parameter. Every spike was then recorded as a set of parameters reflecting its channel identity, location, time, amplitude and shape.

A single mechanical motion can produce spike signals in several channels. To count the real number of "independent" events, the globalisation procedure is applied. It groups coincident spikes (the criterion \pm 1 sampling rate was used) detected inside the same magnet element (aperture or section) into a "global spike". Each of them can be associated to a common mechanical event. So the number of "global spikes" reflects the real global mechanical activity inside the magnet. The second part of the globalisation procedure applied to QA channels is to consider the possible sequential propagation of the mechanical motion from initial to neighbouring sections.

As a result every registered spike obtains a "global spike" number for identification. It is then possible to analyse the signature of every mechanical motion and to characterise its features. All results are finally stored as files and are ready for statistical analysis which can be performed with different software.

\section{RESULTS AND ANALYSIS}

\section{A. Example of spike counting and axial localisation given by a first software version}

An example of output which can be obtained from the data treatment developed for this study, is given in Fig. 4. In the upper part, the number of spikes detected by the QuenchAntenna and the Voltage-Taps techniques are reported as a function of time during the powering of a prototype magnet.

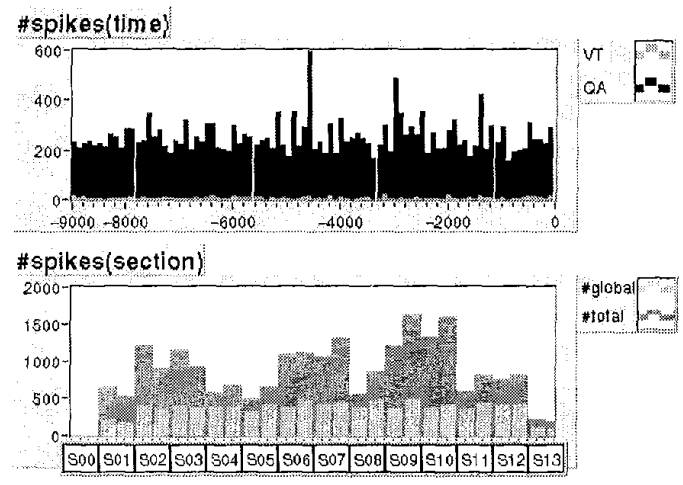

Fig. 4. Example of spikes distribution of all amplitudes in time (scale in $\mathrm{ms}$ ) and in space (scale in section split for both apertures).

It can be seen that the QA detected many more spikes than VT mainly because the coil compensation gives a better signal to noise ratio. The number of spikes detected in each section for both apertures is also reported in Fig.4. Note that both extremities of the magnet (section S01 and S13) are only covered by about $17 \%$ of one section of the shafts. As a consequence, absolute number of spikes obtained for both these sections should be corrected before being compared to the numbers obtained for all other sections ( $\mathrm{S} 02$ to $\mathrm{S} 12$ ). This treatment can be repeated for different pre-selected amplitudes of spikes.

The total number of independent spikes which were detected with QA for $9 \mathrm{~s}$ before the quench for both apertures (Fig. 4) is around 9250. This gives an average frequency of occurrence of events inside the whole twin dipole of around $1 \mathrm{kHz}$.

\section{B. Global characterisation : Number of spikes versus amplitude}

The occurrence of spikes was investigated $9 \mathrm{~s}$ before the first quench of three full-scale LHC magnet prototypes. The field value reached by each magnet is reported in Table $\mathrm{I}$.

TABLE I

VALUE OF THE MAGNETIC FIELD AT THE FIRST QUENCH FOR THREE LHC FULL-SCALE MAGNET PROTOTYPES.

\begin{tabular}{lccc}
\hline Magnet Name & MBP1A1 & MBP2N2 & MBP2O1 \\
Field at the $1^{\text {st }}$ quench (T) & 8.07 & 7.46 & 8.23 \\
\hline
\end{tabular}

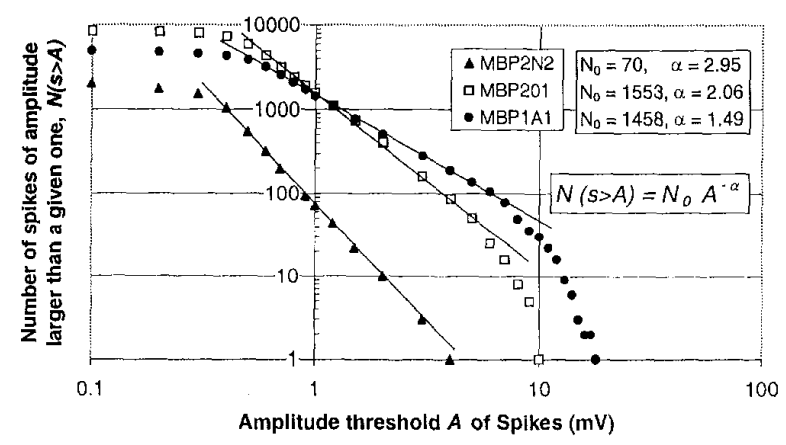

Fig. 5. Log-log plot of the number of spikes of amplitude larger than a reference one, recorded $9 s$ before the first quench of three different fullscale LHC prototypes.

The relation between the number of spikes and the amplitude can be seen on log-log scale in Fig. 5. More precisely, the cumulative statistic has been used to plot the number of spikes with amplitude $s$ larger than a reference one, $N(s>A)$. Apart from cut-off at low and high spike amplitude, the data can be well fitted with the power law

$$
N(s>A)=N_{0} A^{-\alpha} \text {. }
$$

This parameterisation puts forward two main parameters $N_{0}$ and $\alpha$ reflecting the global behaviour of the mechanical activity of the magnet. The correlation between results given in Table $\mathrm{I}$ and in Fig.5 indicates that $N_{0}$ and $\alpha$ should both be as high as possible to optimise the quench performance. Of course such a conclusion should be confirmed with more data. Results already obtained indicate that a superconducting 
magnet can exhibit good quench performance if it can absorb the mechanical load with many micro-scale conductor motions uniformly distributed. This proceeds to a fragmentation of the energy release and to a uniform compaction of the winding without local accumulation of stress. Indeed, any local over-stress can provoke a spike with a sufficient amplitude to trigger a quench. Moreover a magnet with good quench performance should also have the number of spikes with high amplitude as low as possible. This behaviour is expressed by a high value of the parameter $\alpha$ in (1).

The problem of occurrence of spikes inside superconducting magnets looks like the one of earthquakes. Indeed, the expression (1) used to interpret data for example, is close to the Gutenberg-Richter law [4] except that the latter consider the energy released rather than the amplitude of events. Moreover, in this law, the exponent $\alpha$ is directly connected to the mechanical properties of the crust of the earth and the development of this analogy may be worthwhile.

\section{EXPECTATIONS FROM THIS STATISTICAL ANALYSIS}

\section{A. Practical aspects}

Diagnosis and Prediction of quench performance of $L H C$ magnets

The statistic of conductor motions (spikes) before the quench of the magnet contains the information concerning the ability of the coil structure to absorb the mechanical load. As an indication, the Lorentz force gives a resultant around $1.7 \mathrm{MN} / \mathrm{m}$ at nominal field in the first coil quadrant of LHC dipoles.

The data shown in Fig. 4 allow the location of the active parts as a function of the spike amplitudes. This indicates the mechanically weak points inside the superconducting magnet. Such a diagnosis is particularly important for the quality control because it can give a precise feedback to the manufacturer.

The results in Fig. 5 indicate that the quench performance of a given magnet can be qualitatively established before the first quench. More results are needed to fully validate such a diagnosis method and a systematic investigation of all prototypes is underway. Moreover the next step of this study will be to make a quantitative prediction of the field value for which a quench is expected (within a given uncertainty). To reach this target, the number of spikes should be counted as a function of time throughout the whole powering period of the magnet. The use of special counters already developed for particle detection is under study. The particular interest of this diagnosis method will appear during the series test of all LHC main ring magnets for which the number of quenches will be restricted.

The global overview of the mechanical activity of a superconducting magnet can be immediately seen in a log-log plot of the type shown in Fig. 5. The parameterisation with a power law puts forward two main parameters $N_{0}$ and $\alpha$, both should be as high as possible to optimise the quench performance. This approach will be repeated at more local levels (section by section for example) and a better understanding of the quench behaviour of LHC magnets [1] is expected.

\section{B. Fundamental aspects}

The statistical analysis of spike distribution will bring useful information for a better understanding of the training behaviour of superconducting magnets.

Moreover, the analogy already mentioned with earthquake physics is quite illustrating of the broader extension which should be given to this work. Moreover, recent theoretical developments in this field try to introduce a universal concept of "Fractals and self-organized criticality" [5] to explain the power law occurrence in a lot of natural phenomena. The study of the mechanical activity of global and complex systems coming from different manufacturers will give a unique opportunity to test this type of approach. In that sense, the LHC superconducting dipoles can be considered as a model to study the dynamics of complex systems and their behaviour can bring useful information in the general field of hazard prediction.

\section{V.CONCLUSIONS}

All results presented here come from a first version of a software development which extracts, counts and localises electromagnetic signals resulting from conductor motions inside a superconducting magnet. Results obtained show that this diagnosis method is very promising at least for the quality control of the fabrication of the LHC main ring magnets during series tests at low-temperature.

\section{ACKNOWLEDGMENT}

The authors wish to thank P. Sievers for his constant support, J. Billan and his team for the excellent work done on ceramic shafts, G. D'Angelo and his colleagues for devotion in testing of superconducting magnets.

\section{REFERENCES}

[1] L. Bottura, P. Pugnat, A. Siemko, J. Vlogaert, and C. Wyss "Performance of the LHC Final Design Full Scale Superconducting Dipole Prototypes", Appl. Sup. Conf., September 2000, submitted for publication,

[2] D. Leroy, J. Krzywinski, V. Remondino, L. Walckiers and R. Wolf, "Quench observation in LHC superconducting one meter long dipole models by field perturbation measurements", IEEE Trans. Appl. Sup., Vol. 3, pp. 781-784, 1993.

[3] J. Billan, L. Bottura, M. Buzio, G. D'Angelo, G. Deferne, O. Dunkel P. Legrand, A. Rijllart, A. Siemko, P. Sievers, S. Schloss and L. Walckiers, "Twin rotating coils for cold magnetic measurements of $15 \mathrm{~m}$ long LHC dipoles", IEEE Trans. Appl. Sup., Vol. 10, No 1, pp. 1422-1426, March 2000

[4] B. Gutenberg and C. F. Richter, "Earthquake magnitude, intensity, energy and acceleration", Bull. Seism. Soc. Am., Vol. 46, pp. 105-143, 1956.

[5] P. Back, C. Tang and K. Wiesenfeld, "Self-organized Criticality," Phys. Rev. A, Vol. 38, No 1, pp. 364-374, 1988 